\title{
Etude comparative de la productivité de repousses et de la capacité de charge des hémicryptophytes soumises aux feux de végétation dans les parcelles irriguée et non irriguées dans la Réserve Transfrontalière de Biosphère (RTB) du W - Benin
}

\author{
B. Doris AHOUANGAN ${ }^{1 *}$, Marcel HOUINATO ${ }^{2}$, Bernard AHAMIDE ${ }^{2}$, Euloge \\ AGBOSSOU $^{2}$ et Brice SINSIN ${ }^{2}$
}

${ }^{1}$ Spécialiste en gestion des ressources naturelles, BP. 1529 Calavi, Bénin.

${ }^{2}$ Faculté des Sciences Agronomiques, Université d'Abomey-Calavi, BP. 526 Cotonou, Bénin.

* Corresponding authors, E-mail: abnice84@yahoo.fr, Tél: + (229)97337835

\section{RESUME}

La disponibilité des fourrages dans le secteur soudano-sahélien est remarquablement faible en saison sèche, notamment dans La Réserve Transfrontalière de Biosphère (RTB-W Bénin) qui, de par sa position géographique, est de plus en plus menacée par la sahélisation. La présente étude a pour but de contribuer à l'amélioration de la productivité du fourrage vert en saison sèche pour les herbivores sauvages du parc W. Elle a porté sur le pâturage à Andropogon gayanus var bisquamulatus et Vitellaria paradoxa aux chutes de Koudou. Au niveau de ce pâturage, une parcelle irriguée (feu précoce à cycle répétitif et apport d'eau) et deux parcelles non irriguées, l'une ayant subi un traitement de feu précoce à cycle rotatif et l'autre ayant subi un traitement de feu précoce à cycle répétitif ont été définies. Les résultats de cette étude ont révélé que le biovolume varie de $82,4 \mathrm{~m}^{3} /$ ha à $953,0 \mathrm{~m}^{3} / \mathrm{h}$. La plus grande valeur est obtenue sur la parcelle irriguée $\left(953,0 \mathrm{~m}^{3} / \mathrm{ha}\right)$ et la plus faible sur la parcelle non irriguée à cycle rotatif $\left(82,4 \mathrm{~m}^{3} / \mathrm{ha}\right)$. La phytomasse maximale obtenue en fin de période d'expérimentation sur la parcelle irriguée est de 617,17 $\pm 269,01 \mathrm{~kg} \mathrm{MS} / \mathrm{ha}$. Cette phytomasse est plus élevée que celle enregistrée dans les parcelles sans apport d'eau : 41,71 $\pm 10,67 \mathrm{~kg}$ MS/ha pour le cycle rotatif et $201,06 \pm 15,82 \mathrm{~kg}$ MS/ha pour le cycle répétitif. La demande en terre équivalente sur la parcelle irriguée est de $0,15 \mathrm{ha} / \mathrm{UBT}$ en saison sèche, et il faut 3,06 à 15 fois plus de terre pour nourrir une (1) unité bovine tropicale (UBT) en saison sèche en absence d'apport d'eau.

(C) 2010 International Formulae Group. All rights reserved

Mots clés: Pâturages naturels, productivité, biovolume, parc W-Bénin, chutes de Koudou.

\section{INTRODUCTION}

La Réserve Transfrontalière de Biosphère RTB-W (Bénin), l'un des plus grands écosystèmes protégés des savanes d'Afrique constitue, l'une des plus importantes réserves faunique et floristique du Bénin. Pendant plusieurs décennies, ce parc a subi de fortes pressions anthropiques et les dégradations des habitats naturels menacent plusieurs espèces d'herbivores qui perdent leurs ressources alimentaires préférentielles (Sinsin et al., 2005).

L'alimentation du bétail en saison pluvieuse ne pose aucun problème en raison 
de l'abondance des graminées en cette période. Lorsque s'installe la saison sèche avec les feux de végétation, on obtient une bonne repousse à partir des plateaux de tallage des hémicryptophytes qui dominent généralement les autres types biologiques présents (Lejoly et Sinsin, 1991). Ces jeunes repousses de graminées permettent aux animaux de s'alimenter pendant quelques semaines. Une fois ces jeunes repousses de graminées totalement consommées, le problème de l'alimentation de la faune sauvage devient alors de plus en plus crucial au fur et à mesure que la saison sèche perdure. Ainsi, dans le parc $\mathrm{W}$, il se pose un véritable problème de disponibilité en fourrage vert surtout en cette saison qui s'étale sur une longue période au Nord du Bénin. Alors, le maintien de la croissance des graminées vivaces et l'accroissement de leur capacité de régénération pourrait constituer une des solutions adéquates pour la disponibilité en fourrage au cours de la saison sèche. Pour ce faire, la mise en place d'un système d'irrigation s'avère nécessaire. Cette étude a pour objectif d'évaluer la productivité comparée de repousses des hémicryptophytes dans des placeaux soumis aux feux précoces sous micro-irrigation ou non en saison sèche dans la RTB /W.

\section{MATERIEL ET METHODES}

Sinsin et al. (2004) ont identifié les pâturages de la RTB-W (Bénin) soumises chaque année à des essais de feux de végétation sur le tapis graminéen de cette réserve. La présente étude s'est déroulée sur le pâturage à Andropogon gayanus et Vitellaria paradoxa. Cette phytocénose se retrouve dans les savanes arborées sur plateau des secteurs de Sampéto et des chutes de Koudou. Cette homogénéité floristique a permis une comparaison effective et plus aisée des résultats obtenus sur les trois (03) parcelles d'expérimentation.

\section{Milieu d'étude}

La Réserve Transfrontalière de Biosphère $(\mathrm{RTB})$ du W/Bénin est située à l'extrême nord-ouest du Bénin. Elle est comprise entre $11^{\circ} 20^{\prime}$ et $12^{\circ} 23^{\prime}$ Nord et $2^{\circ} 04^{\prime}$ et $3^{\circ} 05^{\prime}$ Est (Figure 1).

De part ses importants écosystèmes, sa diversité biologique et des efforts de gestion déployés par les divers Etats, le Parc National du W (PNW) a été classé Réserve Transfrontalière de Biosphère (RTB) du W depuis 2001 et couvre une superficie de 762.438 ha (partie béninoise).

Le milieu est sous l'influence du climat tropical du type soudano-sahélien, caractérisé par deux grandes saisons : une saison sèche qui s'étend d'octobre à avril et une saison des pluies qui s'étend de mai à septembre. Les températures moyennes annuelles varient de $26^{\circ} \mathrm{C}$ à $33^{\circ} \mathrm{C}$. L'insolation est de 2900 heures en moyenne dans l'année. La période la plus ensoleillée va d'octobre à juin et la moins ensoleillée de juillet à septembre. La moyenne pluviométrique enregistrée au cours des dix dernières années est de 1053,1 $\mathrm{mm}$ de pluie avec des hauteurs maximales au cours des mois de juillet et d'août (Figure 2).

\section{Détermination des périodes de sécheresse}

La formule énoncée ci-dessous par MALTON permet de déterminer les périodes de sécheresse où l'apport d'eau devient indispensable pour la survie des plantes pour la période d'étude.

Si I $>20$ on a une période humide

Si I $<20$ on a une période sèche

$\mathrm{La}$ RBT/W du Niger comporte plusieurs types de sols; il s'agit des sols ferrugineux tropicaux appauvris, fortement concrétionnés avec une surface cuirassée occupée par une végétation arbustive et herbacée dont la litière est rapidement décomposée et minéralisée ou le plus souvent brûlée, des sols ferrugineux tropicaux, sabloargileux ou limono-argileux, noirs, épais et argileux (souvent avec une hydromorphie) qui se révèlent très fertiles.

Les rudes conditions écologiques du milieu caractérisées par l'avancée du Sahel, associées aux pratiques annuelles des feux de végétation, ont favorisé la prépondérance des savanes arbustive et herbeuse. Dans ces 
savanes, les feux consument tout le fourrage disponible, créant de ce fait un sérieux problème à l'alimentation des herbivores en saison sèche.

\section{Dispositif expérimental}

Un placeau de longueur $25 \mathrm{~m}$ et de largeur $20 \mathrm{~m}$, soit d'une superficie de $500 \mathrm{~m}^{2}$ a été installé à titre d'essai dans la zone des chutes de Koudou. Pour irriguer ce placeau, des rampes ont été disposées sur toute la longueur du placeau à intervalle de $1 \mathrm{~m}$. Sur chaque rampe des goutteurs ont été installés à chaque $30 \mathrm{~cm}$. Un arrosage quotidien d'une heure est fait pour irriguer le placeau.

Il est important de rappeler que ce système a été enterré à $50 \mathrm{~cm}$ dans le sol. En effet, selon Dautrebande (1983), l'objectif de l'irrigation goutte-à-goutte est d'amener l'eau non seulement localement et uniquement dans la zone racinaire utile mais aussi d'assurer cet apport à une fréquence élevée, donc à faible débit. Les goutteurs sont conçus pour produire autour du point d'émission une infiltration tridimensionnelle développant «bulbe d'humidité » tridimensionnel limitée en principe à la zone active de développement racinaire.

L'évaluation de l'apport d'eau par l'irrigation goutte-à-goutte est fonction de l'uniformité de la distribution des goutteurs. La détermination de cette uniformité a consisté à évaluer les quantités d'eau libérées par l'ensemble des goutteurs échantillonnés à chaque prélèvement, et à étudier leur variabilité dans l'espace et dans le temps. A cet effet, seize (16) points de mesure répartis sur quatre (04) rampes ont été choisis de façon à couvrir la totalité de la superficie irriguée.

La $1^{\text {ère }}$ rampe choisie correspond à celle située au $1 / 5$ en amont de la porte rampe, la $2^{\text {ème }}$ au $2 / 5$, la $3^{\text {ème }}$ au $3 / 5$ et la $4^{\text {ème }}$ au $1 / 5$ en aval de ladite porte rampe.

Sur chacune de ces quatre (04) rampes, quatre (04) goutteurs ont été choisis suivant le même principe que celui précédemment utilisé dans le choix des rampes.

Au niveau de ces seize (16) points ou goutteurs ainsi identifiés, la quantité d'eau sortant a été mesurée tous les 10 jours pendant 5 minutes. Les mesures effectuées ont permis à partir des calculs du débit de l'eau d'établir les tables d'analyse nécessaires à l'évaluation de l'efficacité du système d'irrigation $C f$. Schéma du dispositif d'irrigation (Figure 3).

\section{Choix des dispositifs}

Dans le souci d'obtenir de bons éléments de comparaison, deux parcelles non irriguées considérées comme des témoins et une parcelle irriguée ont été pris en compte. Le choix des parcelles a été fait de façon aléatoire simple. Les différents types de traitement subi par ces parcelles sont présentés dans le Tableau 1.

Il est à remarquer que la parcelle non irriguée à cycle rotatif correspond à une des portions de terre de la grande parcelle représentée à la Figure 4 ci-dessous. Elle a subi chaque année différents traitements de feux (Mise en défens, Feu de contre-saison, Feu tardif, Feu précoce) dont l'effet rotatif s'applique suivant le sens contraire des aiguilles d'une montre. La parcelle non irriguée à cycle rotatif a subi un feu précoce pendant l'année d'expérimentation.

\section{Evaluation de productivité des parcours}

La détermination de la phytomasse produite est réalisée par la méthode des coupes rases au moyen du sécateur $\mathrm{du}$ jardinier dans les trois parcelles de longueur $25 \mathrm{~m}$ et de largeur $20 \mathrm{~m}$, soit une superficie de $500 \mathrm{~m}^{2}$ chacun :

- Une parcelle irriguée installée à titre d'essai dans la zone des chutes de koudou ;

- Deux (02) parcelles non irriguées dont le premier à cycle rotatif et le second à cycle répétitif.

Dans chaque parcelle, trois (03) placettes de $5 \mathrm{~m}$ x $5 \mathrm{~m}$ sont choisies sur la diagonale pour tenir compte de la variabilité au sein de la parcelle. La phytomasse des hémicryptophytes ainsi récoltée a été triée en deux catégories : les graminées vivaces et les autres espèces. Les poids frais ont été obtenus sur le terrain à l'aide de pesons à ressort appropriés au niveau des placettes. Un 
échantillon de $120 \mathrm{~g}$ du matériel végétal frais de chaque lot a été prélevé au niveau de chaque placeau dans un sachet de productivité pour la détermination de la matière sèche. Un pré séchage a été effectué sur le terrain. Les poids secs de tous les échantillons récoltés pour l'estimation de la phytomasse ont été notés après séchage à l'étuve à $105^{\circ} \mathrm{C}$ jusqu'à poids constant au Laboratoire de Zootechnie de la Faculté des Sciences Agronomiques du Benin. En raison de l'apport quotidien d'eau sous forme de micro-irrigation en saison sèche et afin de suivre de façon rapprochée les fluctuations de repousse, le rythme de coupe est de 15 jours, contrairement à celui préconisé par la littérature qui est d'au moins 30 jours pour les repousses de saison sèche.

Les capacités de charge sont calculées selon la méthode de Boudet (1991) sur la base de la phytomasse consommable par unité bovine tropicale (UBT). La fraction consommable considérée est de $1 / 3$ de la phytomasse totale afin de maintenir une couverture herbacée pouvant protéger le sol contre tout facteur de dégradation.

Les mesures de hauteur, la circonférence du plateau de tallage, la densité des touffes ont eu lieu à l'intérieur des placettes identiques et adjacentes aux précédents. Ces mesures sont prises avant la coupe de phytomasse et ont permis d'apprécier le pouvoir de régénération des hémicryptophytes et de suivre leur évolution durant la période de l'expérimentation.

Le biovolume est le produit du recouvrement par la hauteur, prise de la base des tiges à la dernière feuille (Descoings, 1976).

Les analyses statistiques ont porté sur l'analyse de variance avec utilisation du test de Newman-Keuls et la corrélation.

\section{RESULTATS}

\section{Productivité de biomasse et capacité de charge}

Les données de productivité de phytomasse maximale obtenues en fin d'expérimentation sur les trois parcelles au cours de la période sèche et leur capacité de charge correspondante sont présentées dans le Tableau 2.

La plus forte productivité $(617,17 \pm$ 269,01 kg MS/ha) a été obtenue sur la parcelle irriguée tandis que la plus faible sur la parcelle non irriguée à cycle rotatif $(41,71 \pm$ 10,67 kg MS/ha).

Par ailleurs, la valeur de la phytomasse produite sur l'ensemble des parcelles d'expérimentation est en moyenne de 286,64 $\pm 297,12 \mathrm{~kg} \mathrm{MS} / \mathrm{ha}$.

L'analyse de variance révèle une différence significative entre les valeurs de phytomasse obtenues pour les différentes parcelles $(p=0,0228)$. Cette variation confirme les tendances observées et montre l'impact positif de l'irrigation et du feu précoce sur la croissance de la productivité de biomasse au cours de la saison sèche. L'effet de l'irrigation sur la vitesse de production des hémicryptophytes est cependant plus remarquable que celui du feu.

La capacité de charge de la parcelle irriguée est plus grande (6,58 $\pm 2,87$ UBT/ha/5 mois) que celle des deux autres parcelles de traitement. La valeur la plus faible est obtenue sur la parcelle non irriguée à cycle rotatif $(0,44 \pm 0,11 \mathrm{UBT} / \mathrm{ha} / 5$ mois $)$. Cette situation justifie le fait que l'apport d'eau est un facteur d'accroissement de la capacité de charge des zones de pâture. De plus, la demande en Terre Equivalente correspondante a montré qu'il faut au moins 0,15 ha pour satisfaire un (1) UBT pendant 5 mois de saison sèche sous l'hypothèse d'apport d'eau sous forme d'irrigation goutte à goutte. Elle est respectivement de 2,25 ha et 0,46 ha pour les parcelles non irriguées à cycle rotatif et à cycle répétitif (Tableau 2). Cela confirme l'importance de l'irrigation dans l'augmentation de la productivité des fourrages disponibles aux herbivores en période sèche.

\section{Régression de la phytomasse et quantité totale d'eau apportée}

La distribution de l'eau étant uniforme sur la parcelle irriguée, les variations de la quantité totale d'eau apportée expliquent à 
$86 \%$ les variations de la quantité totale de phytomasse produite $(\mathrm{p}=0,007)$. Le couple (phytomasse, quantité d'eau) étudié sur toute la période d'étude au niveau de la parcelle irriguée est assez bien corrélé avec un coefficient de détermination de 0,87 . L'apport d'eau pourrait donc être plus bénéfique à l'augmentation de disponibilité de fourrages en saison sèche.

\section{Biovolume des hémicryptophytes}

La Figure 6 présente l'évolution du biovolume des hémicryptophytes au niveau des différentes parcelles au cours de la saison sèche.

L'analyse de la Figure 6 montre que le biovolume est plus élevé sur la parcelle irriguée et sur la parcelle non irriguée à cycle répétitif que sur la parcelle non irriguée à cycle rotatif qui est presque constante de mimars à mis-avril. Il ressort alors que les valeurs du biovolume au cours de la période expérimentale sont globalement plus élevées au niveau de la parcelle irriguée $\left(953 \mathrm{~m}^{3} / \mathrm{ha}\right)$ et de la parcelle non irriguée à cycle répétitif $\left(908,4 \mathrm{~m}^{3} / \mathrm{ha}\right)$ que sur la parcelle non irriguée à cycle rotatif $\left(82,4 \mathrm{~m}^{3} / \mathrm{ha}\right)$. Les plus grandes valeurs de biovolume sont obtenues sur la parcelle irriguée. Cette dominance s'expliquerait, d'une part, par l'effet positif de l'apport d'eau sur la parcelle irriguée et d'autre part, par l'impact du feu précoce appliqué à la parcelle non irriguée à cycle répétitif.

L'analyse de variance révèle une différence significative entre les biovolumes des trois parcelles expérimentales $(\mathrm{p}=$ 0,0001). Cette différence observée au niveau du biovolume correspond à celles de la hauteur et du recouvrement des hémicryptophytes.

En outre, l'étude de la relation entre phytomasse et biovolume total montre une forte corrélation avec un coefficient de corrélation $(\mathrm{r}=0,831)$ significatif $(\mathrm{p}=$ 0,0001). Cette tendance pourrait s'expliquer par le fait que la phytomasse et le biovolume sont deux paramètres caractéristiques de productivité des hémicryptophytes dont la régénération en saison sèche est fortement liée à l'apport d'eau et dans une moindre mesure, par l'application du feu précoce.

\section{Densité de touffes des hémicryptophytes}

$\mathrm{Au}$ cours des trois premières observations du 30 janvier au 01 mars, les valeurs de densité de touffes obtenues (Figure 7) sur les trois parcelles expérimentales sont restées sensiblement égales entre elles mais décroissantes.

A partir de mi-mars, la densité de touffes au niveau de la parcelle irriguée est restée en moyenne supérieure à celle des parcelles non irriguées durant le reste de la période expérimentale.

L'analyse de variance révèle qu'il existe une différence significative entre les densités de touffes d'hémicryptophytes au niveau des trois parcelles $(\mathrm{p}=0,0038)$. Ces observations traduisent l'effet de l'apport d'eau sur la croissance des hémicryptophytes.

Par ailleurs, l'étude de corrélation faite sur la parcelle irriguée $(r=0,21 ; p=0,38)$, sur la parcelle non irriguée à cycle rotatif $(\mathrm{r}=$ $-0,003 ; p=0,98)$ et sur parcelle non irriguée à cycle répétitif $(\mathrm{r}=0,22 ; \mathrm{p}=0,37)$ montre de façon globale une absence de différence significative et donc une faible liaison entre nombre de touffes et biovolume totale. La densité des touffes n'a donc pas d'influence sur la croissance du biovolume et l'évolution du biovolume observée au niveau des hémicryptophytes pourrait cependant être due à leur typologie ou espèces présentes sur les parcelles.

La corrélation est moyenne entre la phytomasse et le nombre de touffes, avec $\mathrm{r}=$ 0,521 significatif $(p=0,0001)$. Cela nous permet de dire que la densité des touffes n'a pas une grande influence sur la phytomasse produite.

$$
I=\frac{12 P}{-------}
$$

Avec $\mathrm{P}$ : pluviométrie en $\mathrm{mm}$

$\mathrm{T}$ : température en ${ }^{\circ} \mathrm{C}$

$\mathrm{I}:$ indice de sècheresse 
Tableau 1: Différentes parcelles avec leur traitement.

\begin{tabular}{lll}
\hline Type de parcelles & Dimension & Traitement \\
\hline parcelle irriguée à cycle répétitif & $25 \mathrm{~m} \times 20 \mathrm{~m}$ & Feu précoce + irrigation \\
\hline parcelle non irriguée à cycle rotatif & $25 \mathrm{~m} \times 20 \mathrm{~m}$ & Feu précoce \\
\hline parcelle non irriguée à cycle répétitif & $25 \mathrm{~m}$ x $20 \mathrm{~m}$ & Feu précoce \\
\hline
\end{tabular}

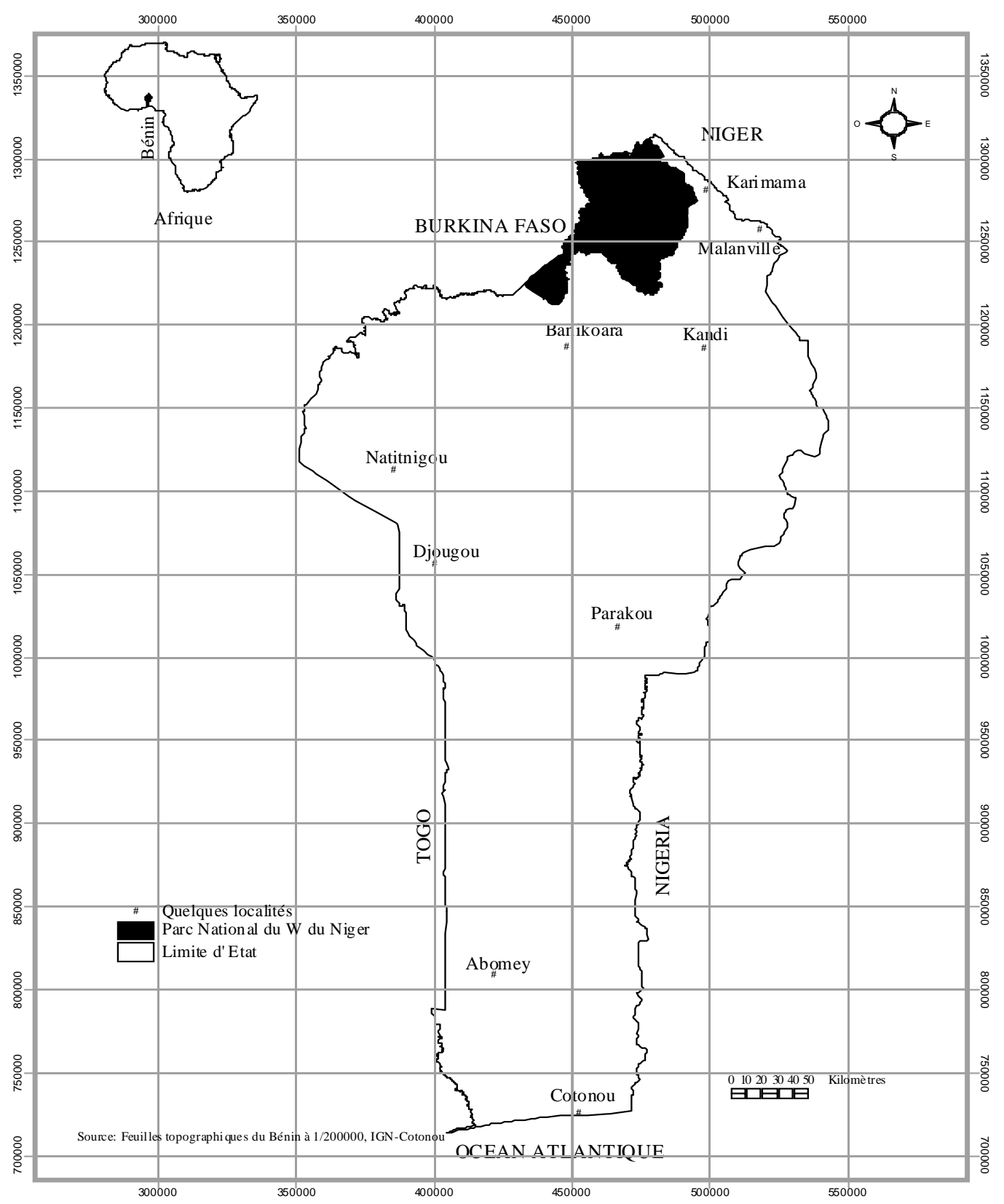

Figure 1 : Localisation de la zone d'étude au Bénin. 
Tableau 2: Phytomasse maximale, capacité de charge et demande en terre équivalente sur les diverses parcelles.

\begin{tabular}{lccc}
\hline Types de parcelle & $\begin{array}{c}\text { Phytomasse } \\
\text { maximale } \\
\text { (kg MS/ha) }\end{array}$ & $\begin{array}{c}\text { Capacité de } \\
\text { charge } \\
\text { (UBT/ha/5 mois) }\end{array}$ & $\begin{array}{c}\text { Demande en Terre } \\
\text { Equivalente } \\
\text { (ha/UBT/5 mois) }\end{array}$ \\
\hline PIR & $617,17 \pm 269,01^{*}$ & $6,58 \pm 2,87$ & 0,15 \\
PNR $_{\mathbf{1}}$ & $41,71 \pm 10,67^{*}$ & $0,44 \pm 0,11$ & 2,25 \\
PNR $_{2}$ & $201,06 \pm 15,82^{*}$ & $2,14 \pm 0,17$ & 0,46 \\
\hline
\end{tabular}

${ }^{*}$ Différence significative $(\mathrm{p}=0,0228)$;

PIR = Parcelle irriguée (feu précoce à cycle répétitif et apport d'eau) ;

PNR1 = Parcelle non irriguée 1 (à cycle rotatif ayant subi un feu précoce) ;

PNR2 = Parcelle non irriguée 2 (à cycle répétitif ayant subi un feu précoce).

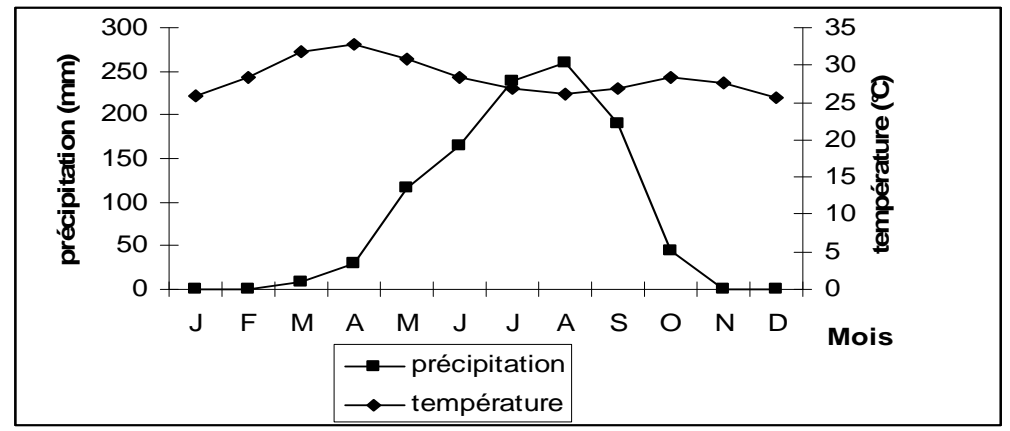

Figure 2 : Diagramme ombrothermique de la RTB/W (moyenne des années 1997-2006).

Source : Données de l'ASECNA Station Météo/ Aérodrome Kandi.

\section{DISCUSSION}

Les pâturages dans la RTB-W Bénin présentent une productivité relativement élevée en saison pluvieuse. Les valeurs de phytomasse maximale obtenues en zone soudano-sahélienne sur le présent site expérimental par Sinsin et al. (2005), varient entre $4200 \pm 3200$ et $5100 \pm 2200 \mathrm{~kg} \mathrm{MS} / \mathrm{ha}$ avec des capacités de charge comprises entre $0,6 \pm 0,5$ et $0,7 \pm 0,3 \mathrm{UBT} / \mathrm{ha} / \mathrm{an}$ selon les groupements végétaux étudiés dans la même zone.

En région soudanienne au BurkinaFaso, les résultats de phytomasses maximales obtenus par Fournier en 1990 (soient de 2000 à $12000 \mathrm{~kg} \mathrm{MS} / \mathrm{ha}$ ) dans les savanes de Nazinga $\left(11^{\circ} 01\right.$ ' N) selon les faciès de végétation et la topographie, corroborent ceux trouvés dans la même région au Bénin. En région soudano-guinéenne des Monts Kouffé, Houinato et al. (2003) ont obtenu des valeurs de phytomasse herbacée variant de 2800 à $7300 \mathrm{~kg} \mathrm{MS} / \mathrm{ha}$.

Suite à l'arrêt de pluies, les feux de brousse passent et consument la quasi-totalité de la biomasse présente. Selon Houinato et al. (2003) dans la zone soudano-guinéenne des monts Kouffé, ces feux emportent 80 à $90 \%$ de la paille laissant stationnellement 10 à $20 \%$ de paille variant entre 135,85 et $459,636 \mathrm{~kg}$ MS/ha qui en général sont insuffisants pour l'alimentation du bétail. On observe une bonne repousse à partir des plateaux de tallage des hémicryptophytes qui représentent le type biologique le plus dominant (supérieur à $60 \%$ dans la zone soudano-sahélienne selon Agbessi (2005)) (Lejoly et Sinsin, 1991). Ces jeunes repousses de graminées permettent aux animaux de s'alimenter pendant quelques semaines. Ceci s'apparente très bien aux résultats obtenus sur les parcelles non irriguées au cours de la saison sèche. 


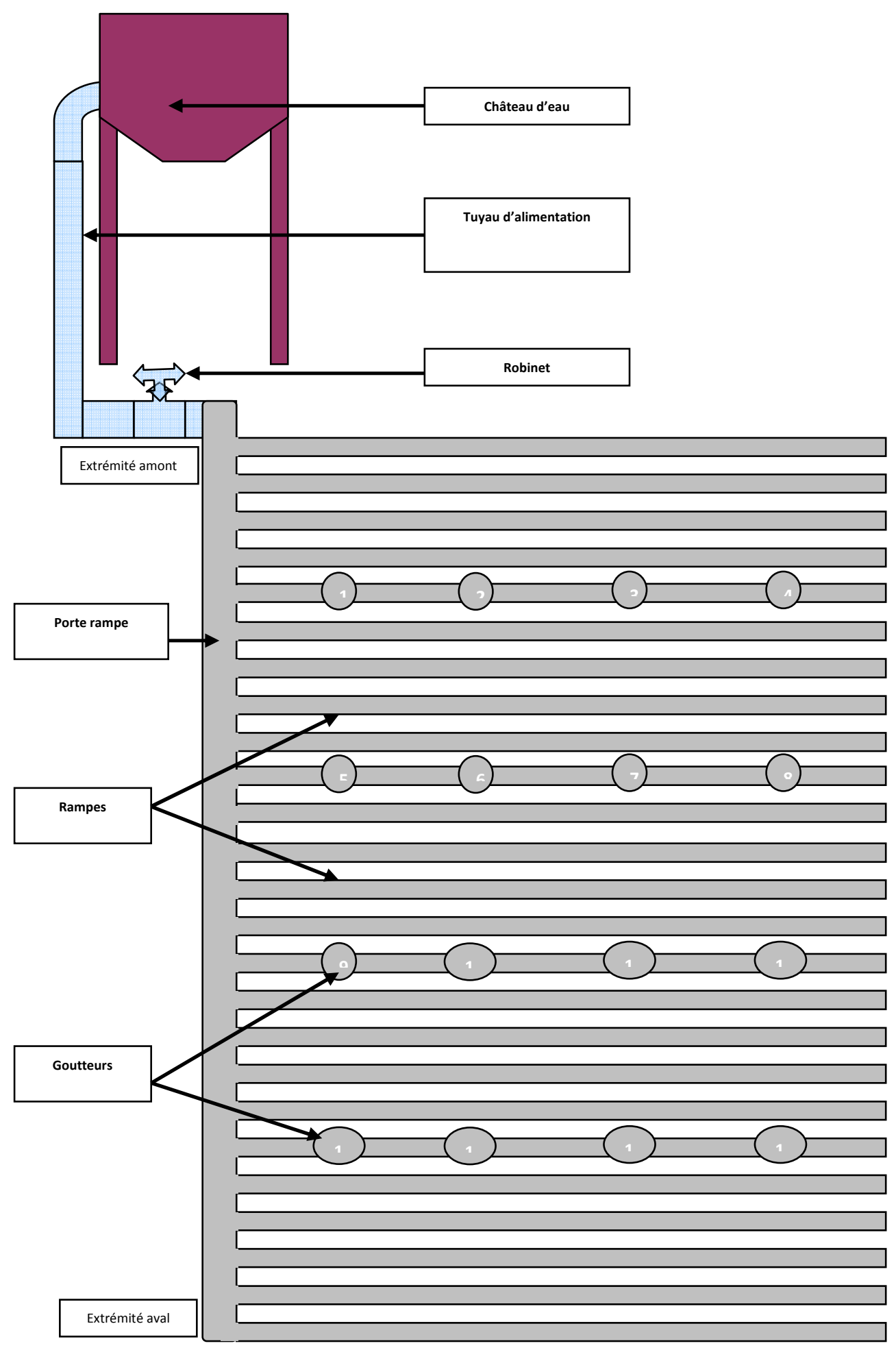

Figure 3 : Schéma du dispositif d'irrigation expérimentale sur le site des chutes de Koudou. 


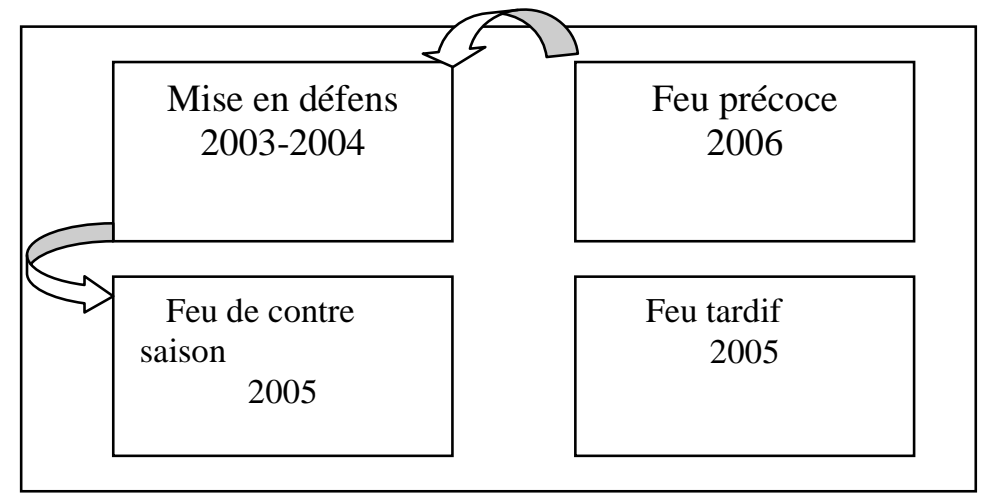

Figure 4: Dispositif expérimental de rotation des feux d'aménagement sur les parcelles ayant servi de témoin. Source : Yaoitcha, 2004.

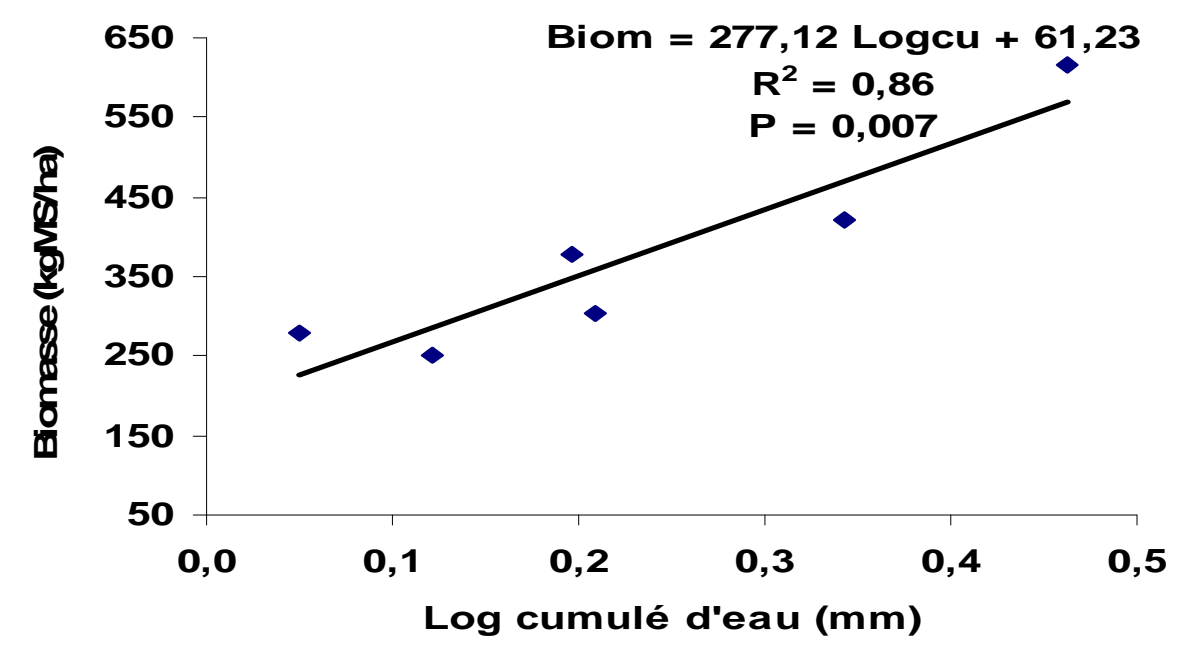

Figure 5: Régression de phytomasse totale produite en fonction de la quantité totale d'eau.

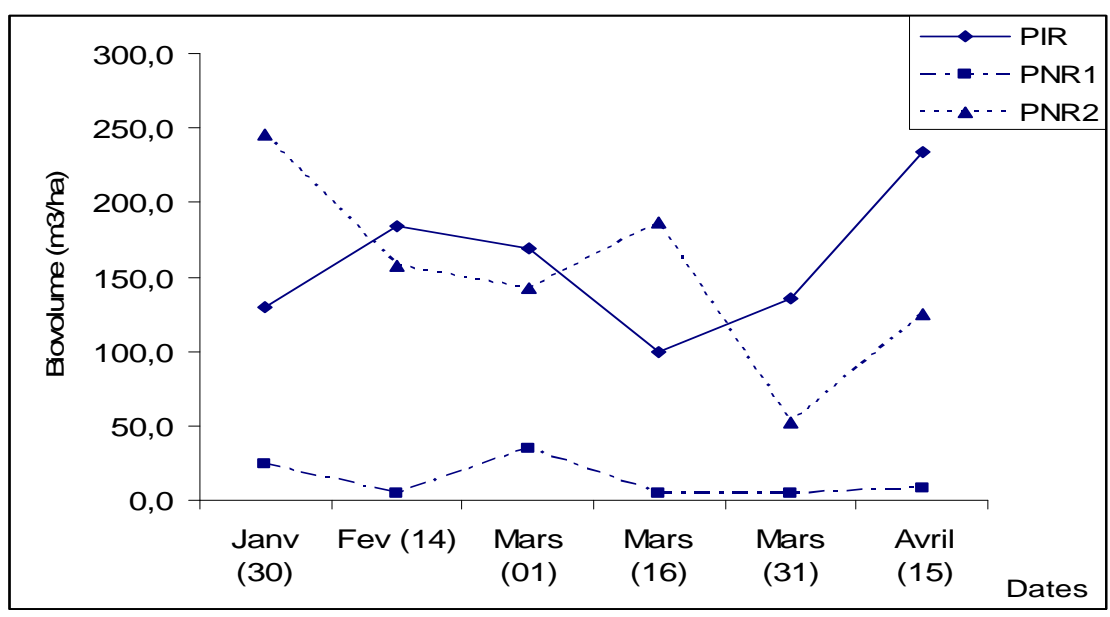

Figure 6: Evolution comparée du biovolume des hémicryptophytes au niveau des différentes parcelles. 


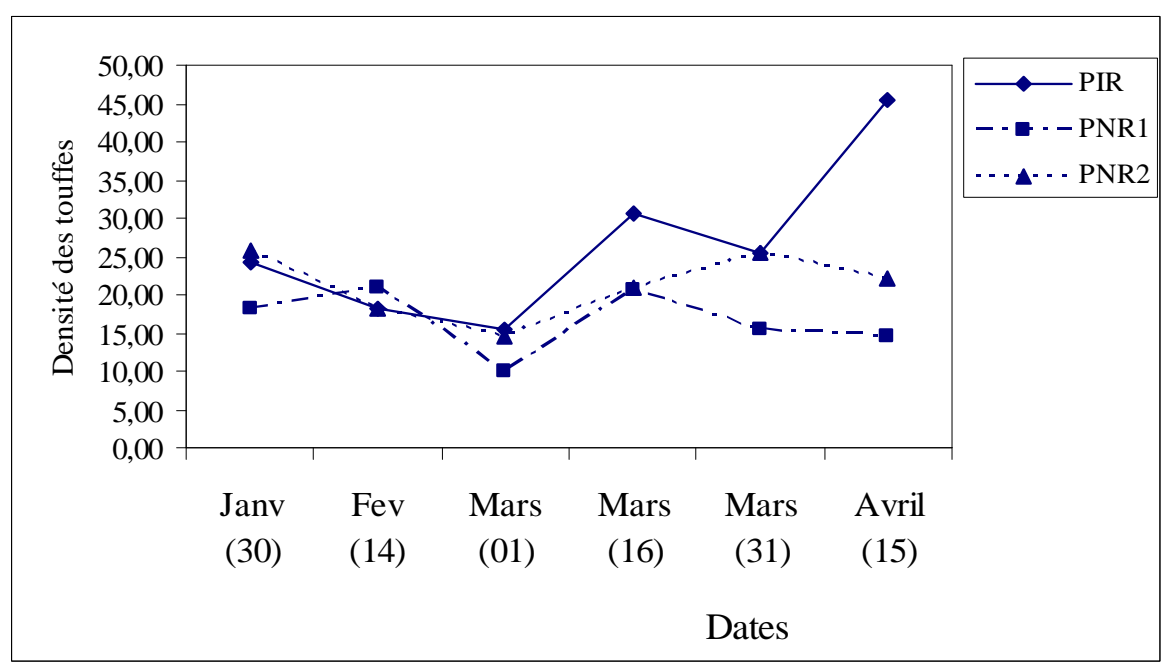

Figure 7 : Variation de l'effectif de touffes d'hémicryptophytes au niveau des différentes parcelles.

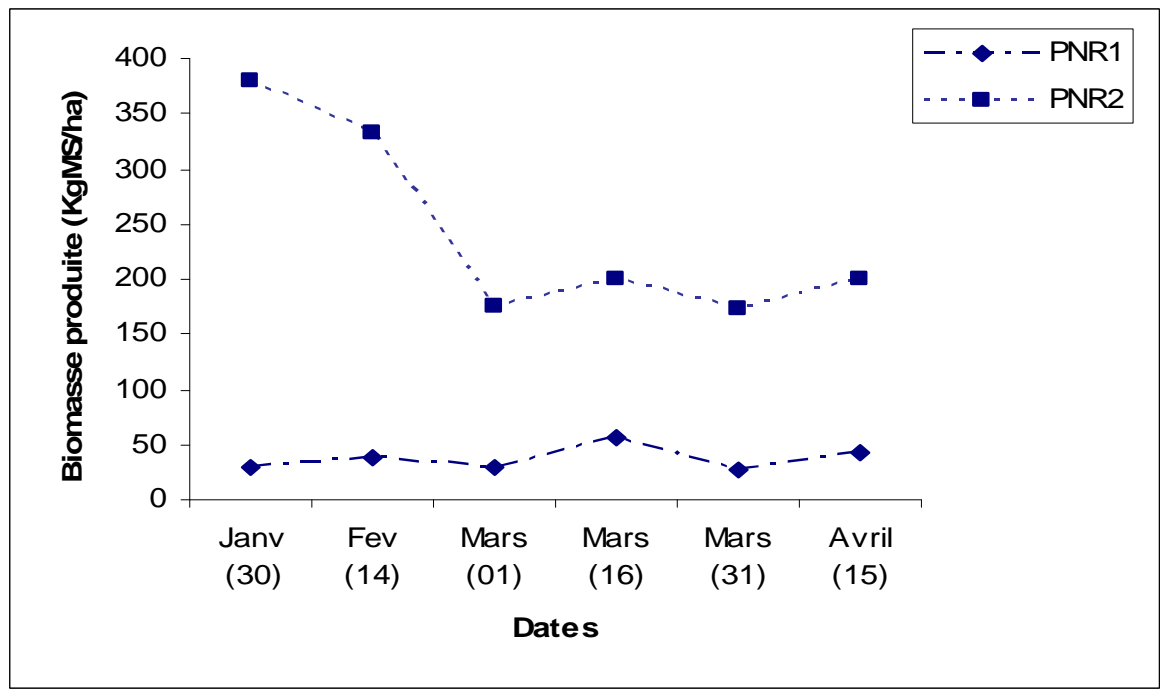

Figure 8: Evolution de la productivité de phytomasse sur les parcelles non irriguées.

En effet, la valeur de phytomasse obtenue en fin d'expérimentation sur la parcelle non irriguée à cycle répétitif est de $201,06 \pm 15,82 \mathrm{~kg}$ M.S/ha. Elle est relativement faible par rapport à celle trouvée en 1989 par Sinsin et al. $(330,95 \pm 52,27 \mathrm{~kg}$ $\mathrm{MS} / \mathrm{ha}$ ) en zone soudanienne pour un même traitement de feu. Cela s'expliquerait par les conditions climatiques peu favorables caractérisant la zone soudano-sahélienne qui représente notre milieu d'étude.

La valeur de phytomasse relativement faible observée au niveau de la parcelle non irriguée à cycle rotatif $(41,71 \pm 10,67 \mathrm{~kg}$ MS/ha) par rapport à la parcelle non irriguée à cycle répétitif (Figure 8) pourrait être justifiée par l'effet des différents traitements de feux auxquels a été soumise cette parcelle les années antérieures. Selon Yaoitcha 
(2004), le feu tardif appliqué à cette même parcelle l'année précédente aurait réduit le recouvrement des hémicryptophytes et donc entraîné une faible phytomasse en raison de son caractère violent qui rend difficile le démarrage des graminées et compromet la repousse des hémicryptophytes.

La valeur de la phytomasse produite sur l'ensemble des parcelles d'expérimentation est en moyenne de 286,64 $\pm 297,12 \mathrm{~kg} \mathrm{MS} / \mathrm{ha}$. Cette valeur est relativement proche de celle obtenue par Sinsin et al. (1989) en période sèche dans la zone soudanienne au nord-est du Bénin et qui s'élève à 330,95 $\pm 52,27 \mathrm{~kg} \mathrm{MS} / \mathrm{ha}$. La parcelle irriguée a donné cependant un meilleur développement d'hémicryptophytes avec une phytomasse consommable de l'ordre de 617,17 $\pm 269,01 \mathrm{~kg}$ MS/ha en fin d'expérimentation. Cette valeur est supérieure à celles obtenues sur la parcelle non irriguée à cycle répétitif et la parcelle non irriguée à cycle rotatif qui sont respectivement de 201,06 $\pm 15,82 \mathrm{~kg}$ M.S/ha et $41,71 \pm 10,67 \mathrm{~kg} \mathrm{MS} / \mathrm{ha})$. Cette situation s'explique par l'apport d'eau sous forme d'irrigation goutte à goutte en saison sèche.

Dans les savanes soudano-guinéenne du Ranch de l'Okpara au nord Bénin, Sinsin et Saïdou (1998) ont prouvé que la productivité des pâturages à Andropogon gayanus peut atteindre $600 \pm 200 \mathrm{~kg} \mathrm{MS} / \mathrm{ha}$ avec 1061,9 $\mathrm{mm}$ de pluie. Ce résultat confirme l'effet de l'apport d'eau sur les graminées pérennes en saison sèche. De plus, les valeurs de phytomasse maximale moyenne $(286,64 \pm 297,12 \mathrm{~kg} \mathrm{MS} / \mathrm{ha})$ produite sur l'ensemble des parcelles d'expérimentation et de la phytomasse maximale sur la parcelle irriguée $(617,17 \pm$ 269,01 kg MS/ha) se retrouvent dans la fourchette des données avancées par UNESCO (1981) (0,2 à 5/tMS/ha) pour les pâturages naturels des zones soudanosahéliennes en saison pluvieuse.

Le couple (phytomasse, quantité d'eau) étudié sur toute la période au niveau de la parcelle irriguée est assez bien corrélé avec un coefficient de détermination de 0,87 . Ce résultat est corroboré par celui de Sinsin (1991) qui a trouvé que le couple (phytomasse, pluie), étudié sur trois années successives dans les pâturages herbacées de la zone soudanienne du Nord Bénin, est fortement corrélé avec un coefficient de corrélation de 0,99 .

$\mathrm{La}$ faible valeur du biovolume enregistrée de façon générale est la conséquence de l'aridité caractérisant la zone d'étude (zone soudano-sahélienne) et de la période expérimentale (saison sèche). La valeur moyenne de biovolume obtenue en expérimentation est d'environ $648 \pm 490,27$ $\mathrm{m}^{3} /$ ha alors qu'elle est de l'ordre $8000 \mathrm{~m}^{3} / \mathrm{ha}$ dans les savanes soudaniennes de plateau en période humide selon Rivas et al. (1985). Cependant l'irrigation a favorisé une élévation relative de cette valeur (soit 953 $\mathrm{m}^{3} / \mathrm{ha}$ ) au dessus de la moyenne. Ce qui montre l'importance et la nécessité de l'apport d'eau pour l'augmentation de la masse fourragère disponible pour les herbivores en période sèche.

Par ailleurs, la similitude entre les valeurs de biovolume obtenues dans la parcelle irriguée et la parcelle non irriguée à cycle répétitif témoigne de l'importance d'un traitement à cycle répétitif par rapport à celui d'un cycle rotatif.

\section{Conclusion}

Au terme de la présente étude dont le but est de contribuer à la conservation des graminées pérennes pour servir de fourrage de contre saison pour les herbivores sauvages dans le parc $\mathrm{W}$, il convient de retenir que l'irrigation constitue l'un des moyens fondamentaux de palier à la faiblesse de la disponibilité de phytomasse pâturable pendant la saison sèche.

En effet, l'apport d'eau influe favorablement sur le développement des hémicryptophytes en période sèche. L'obtention au niveau de la parcelle à cycle répétitif d'une croissance remarquable en biovolume et en phytomasse par rapport à celle de la parcelle à cycle rotatif, rend compte de l'importance et de la nécessité de l'application d'un feu précoce répétitif aux parcours naturels du parc $\mathrm{W}$ pendant la saison sèche. De plus, la différence significative de productivité observée au niveau des parcelles étudiées témoigne de la nécessité d'utiliser l'irrigation comme moyen d'augmentation de la capacité de charge, du maintien et de l'aménagement de ces parcours. 
Une irrigation sur un parcours naturel ayant subi le passage d'un feu précoce répétitif donnerait un meilleur rendement en comparaison à un parcours ayant subi le traitement aux feux rotatifs.

\section{REMERCIEMENTS}

Les auteurs remercient l'Union Européenne, pour avoir financé ce programme de recherche sur la gestion et l'aménagement des parcours naturels par apport d'eau après le passage du feu.

\section{REFERENCES BIBLIOGRAPHIQUES}

Agbessi M. 2005. Productivité et capacité de charge des pâturages naturels de la zone des chutes de Koudou dans la Réserve Transfrontalière de Biosphère du W Bénin. Mémoire du D.I.T, EPAC/UAC, p. 82.

Boudet G. 1991. Pâturages Tropicaux et Cultures Fourragères. Coll. Manuels et Précis d'Elevage $\mathrm{n}^{\circ} 4$, IEMVT. Minist. Coop. ; 266.

Dautrebande S. 1983. La Miro- irrigation. Notes de cours. Faculté des sciences agronomiques de l'Etat. Génie rural II. Université de Gembloux. Cours inédit.

Descoings B. 1976. Approche des formations herbeuses tropicales par la structure de la végétation. Thèse USTL Montpellier, France.

Fournier A. 1990. Phénologie, croissance et production végétales dans quelques savanes d'Afrique de l'Ouest: Variation selon un gradient climatique. Thèse de Doctorat d'Etat, Univers Pierre et Marie Curie Paris 6, p. 312.

Houinato M. Kagone H. Sinsin B. 2003. Seasonal cycle of herbaceous plant phytomass of monts kouffe region in Benin. FSA/UAC Cotonou. Bénin. INERA, Ouagadougou, Burkina Faso.

Lejoly J, Sinsin B. 1991. Structure et valeur pastorale des pâturages soudaniens des bas-fonds dans le Nord-Bénin. Périmètre Nikki- Kalalé : pp. 554-557.

Rivas A, Buldgen A, Compère R. 1985. Etude phytosociologique du plateau andin pampa galéras au Pérou occidental. Tropicultura, 3(1) : 3-13.

Sinsin B, Oloulotan S, Oumorou M. 1989. Les pâturages de saison sèche de la zone soudanienne du Nord Bénin. Rev. Méd. Vét. Pays Tropicaux., 42(2): 283-288.

Sinsin B. 1991. Saison de végétation et production de phytomasse dans les pâturages naturels du Nord-Bénin. In : Actes du séminaire " Influence du climat sur la production des cultures tropicales", Ouagadougou, Burkina Faso. IFS-CTA, Stockholm Suede.

Sinsin B, Saidou A. 1998. Impact des feux contrôlés sur la productivité des pâturages naturels des savanes SoudanoGuinéenne du Ranch de l'OKPARA au Bénin. Annal des Sciences Agronomiques du Bénin, 1: 11-30.

Sinsin B, Houinato M, Bruckmann A, ElHadj Issa A, Yaoitcha AS, Ballo MC, Ahokpè E, Mama A. 2004. Etude sur la biomasse dans la Réserve de Biosphère Transfrontalière du W "gestion des écosystèmes par le feu".

Sinsin B, Houinato M, Bruckmann A, ElHadj Issa A, Yaoitcha AS, Ballo MC, Ahokpè E, Mama A. 2005. Etude sur la biomasse dans la Réserve de Biosphère Transfrontalière du W "gestion des écosystèmes par le feu" Rapport. p. 37.

UNESCO. 1981. Ecosystèmes Pâtures Tropicaux. UNESCO: Paris, France ; 675.

Yaoïtcha AS, 2004. Dynamique des pâturages naturels suivis de 2000 à 2004 sur les fermes d'élevage de Bétécoucou et de Samiondji et l'exploitation pastorale des alentours par les éleveurs bovins riverains. Th. Ing. Agr. FSA/UAC. Abomey-Calavi, Bénin, p. 111. 